\title{
Design and Application of a Virtual Classroom System in Major Courses in Industrial Arts
}

\author{
http://dx.doi.org/10.3991/ijet.v10i5.4796 \\ Yajuan Liu \\ University of Science and Technology Liaoning, Anshan, China
}

\begin{abstract}
The development status and defects of current distance education are pointed out, the theoretical basis and support technology to construct virtual classrooms are demonstrated, and a virtual classroom system under a mutual competition environment is proposed and realized. With the teaching of an industrial arts course as the example, the development and application of a virtual classroom system in a course in industrial arts is discussed. A control experiment was designed, the effect of virtual classroom system was verified, and it was concluded that students in a virtual classroom group can more easily master logical reasoning skills and abstract theoretical knowledge. Overall, the virtual class had a better effect than the real classroom or the distance learning group.
\end{abstract}

Index Terms - distance education; virtual classroom; competition; internet; industrial art major

\section{INTRODUCTION}

The emergence of distance education has broken through the traditional teaching model of intensive teaching systems [1]. However, current distance education mostly uses desktop teaching patterns similar to traditional, i.e. teachers sit in front of the computer, face the camera lens, and remotely give lessons to students with the mouse and keyboard - a non-natural method. Such a method is a simple repetition of teaching and learning activities with a lack of interaction between students and teachers and interaction among students as well as a lack of the learning atmosphere of students in a real classroom [2].

The theory of "three-generation information technology and three-generation distance education" put forward and developed by Canadian scholar Riessen, Dutch scholar Nippe, and British scholar Bez contends that the first generation of distance education originated from "correspondence education" in mid-19th century, which was established based on the development of printing and delivery technologies [3]. The second generation of distance education originated from multimedia teaching and distance education in the mid-20th century, which was established based on audiovisual technologies and mass media such as broadcast television and video and audio recording. The third generation of distance education originated from digital virtual education in late 20th century, which was established based on the development of electronic information communication technology centering on computer multimedia and networks [4]. Weeks et al. examined ASP. NET and Database, which were used to create a dynamic WEB virtual classroom teaching system for a health statistics course [5]. Yang designed a universal learning system that provides a complete learning environment consisting of the information collection center, preference estimation system, forum, and virtual class- room [6]. Fiona and Qing conducted a review of the literature on gamification in educational and learning contexts [7]. Timothy and Kenneth found virtual classrooms must address the unique challenges that digital media create and analyzed how Central Michigan University changed the way it operates to create a modern and comprehensive electronic reserve system [8]. Peng et al. presented a Web teaching system based on virtual classroom management that improved the existing curriculum teaching website for resource sharing so teachers can organize teaching activities and control their teaching schedule in accordance with the requirements of classroom teaching [9].

Because of the late start of China's network construction and its incomplete infrastructure development, domestic distance education, especially virtual education, has gaps in contrast to developed Western countries regarding course type, course content quality, and technical content [10]. This project sought to establish a virtual classroom system under the environment of mutual competition, which can reflect the features of teacher-student interaction and mutual learning and competition among students. It can make a virtual classroom close to the learning environment in a real classroom, allow students to realize mutual competition under non-simultaneous online situations, and have low implementation costs for public acceptance.

\section{THEORETICAL FOUNDATION AND SUPPORTING TECHNOLOGY OF ViRTUAL ClasSROOM SYSTEM}

\section{A. Theory of "cone of experience"}

American audio-visual educationist Edgar Dale put forward the theory of "cone of experience" in his monograph Audio-Visual Teaching Method. He contended that there were primarily three ways for obtaining experience: personal experience, observation and summarization, and abstract generalization. For the virtual classroom, nonlinear multimedia information resources were used to provide learners with a multi-level learning experience such as sensorial overall interaction and scene reappearance to improve learning efficiency and effect.

\section{B. Constructivist learning theory}

Constructivist learning theory emphasizes student orientation and stresses that learners are subjects of information processing and active builders of knowledge rather than passive receivers of knowledge. Learning must be conducted in real situations, a real task that emphasizes the importance of cooperative learning. In constructivism, learners construct and obtain knowledge with the help of others (including teachers and learning partners) by using necessary learning data under a certain situation or background. A virtual classroom system can and must provide 
virtual situations and learning data, guide learners' cooperation and competition, and stimulate the initiative of learners according to the requirements of constructivist.

\section{Humanistic learning theory}

The humanistic learning theory considers that the most useful learning method in modern society is to help learners know how to learn. In the teaching process, the function of the classroom is "teaching how to fish" rather than "giving fish." In the virtual classroom, teachers should acknowledge the significant role of students, provide an atmosphere that promotes learning from the perspective of the students (e.g. examinations, group discussions and pro-active question and answer raising), and allow students to master learning skills and learn to draw inferences about other cases from one instance.

\section{Cooperative learning theory}

Cooperative learning theory, emerging in the 1970 s, is a teaching strategy aimed at promoting learning in which some students cooperate to compete in regard to a given learning objective and in which students are organized for learning in groups or teams.

\section{E. Internet}

The internet is a global network covering the whole world that is formed by mutual connection of a group of general protocols. The internet can realize information exchanging and sharing at a low cost without the limitation of space and make communication in virtual classrooms possible. With the development of science and technology, the internet is becoming mobile, cloud-based, and pervasive and provides a larger development space for virtual classrooms.

\section{F. TCP/IP}

A TCP/IP protocol stack divides the network into five layers: physical layer, data link layer, IP layer, TCP/UDP layer, and application service layer from low to high. The TCP/IP protocol stack provides technical support for data communication between virtual classroom networks.

\section{G. UDP}

TCP and UDP protocols are in the transmission layer of a TCP/IP protocol stack. The TCP can guarantee reliable and error-free data transmission. When multiple data packets are sent, the TCP ensures that all packets will reach the destination and be submitted to the application layer in the correct order. The UDP is a simpler connectionless transmission protocol for the purpose of one-time transmissions. It cannot guarantee consistency between the submission order and sending order of data packets and does not have lost packet retransmission mechanism.

The UDP protocol rather than the TCP protocol is often used for transmission of audio and video files in a virtual classroom system via the internet. When transmitted on the internet, audio and video files are often divided into smaller data packets. Although the loss of one or two data packets in the transmission process is unsatisfactory, it is sustainable. Moreover, modern audio and video encoding algorithms can occasionally recover lost packets and will not cause the reduction of the audio and video quality.

On the other hand, audio and video are very sensitive to time delay. If TCP protocol is used for communication, great time delays will be caused. Even worse in the case of packet loss, the TCP protocol will attempt to resend data packets until it has success, which will produce greater time delays.

\section{H. VoIP}

VoIP, the abbreviation of Voice over IP, refers to voice processing technology based on the internet. This technology compresses voice data coding through compression algorithms, transforms voice data into IP data packets, and transmits them based on an IP network. The receiver connects voice data packets in series and recovers the original voice signal through decompression processing, thus achieving voice transmission on the internet. VoIP can be applied to a virtual classroom system to achieve wide and cheap data transmission, such as voice and video.

\section{Virtual reality}

Virtual reality (VR) technology is a comprehensive technology developed with the integration of comprehensive computer graphics technology, multimedia technology, sensor technology, parallel real-time technology, and artificial intelligence and simulation technology. It creates a three-dimensional image world reflecting the change and interaction of entity objects, in analog form, in real time for users and allows participants to directly participate in the exploration of functions and changes of virtual objects in the environment through the realistic experience of perceptual behaviors such as vision, auditory sense, touch and smell as if they were in a real world. Virtual reality technology applied to virtual classrooms can create a scenic learning environment, present information in multiple dimensions, mobilize utilization of learners' sensory organs, and reproduce natural phenomena that cannot be observed. Additionally, it can change the processing of things in everyday life or help visualize abstract concepts and theories, e.g. demonstrate the internal symmetry of crystals with virtual reality technology. Meanwhile, various virtual laboratories can be established with virtual reality technology. For example, learners can perform various kinds of practices in industrial arts design experiments and do not have to worry about material waste caused by disoperation.

\section{Virtual ClassRoom System DesigN}

\section{A. System function}

This paper studies an intelligent and interactive virtual classroom system with an environment of mutual competition, making the virtual classroom closer to a real classroom and allowing students to achieve mutual competition in non-simultaneous online situations. Students can attend class to learn, race to be the first to answer questions from virtual teachers, and be ranked. Students can raise questions mutually to obtain knowledge and understand the learning situations of other students. They can be ranked through examinations and thereby understand their learning level and gaps with others; and competition with each other to obtain an interest in learning.

\section{B. System architectural design}

\section{1) System architectural design}

The system uses a B/S architectural pattern, i.e. browser/server pattern, as shown in Figure 1. 


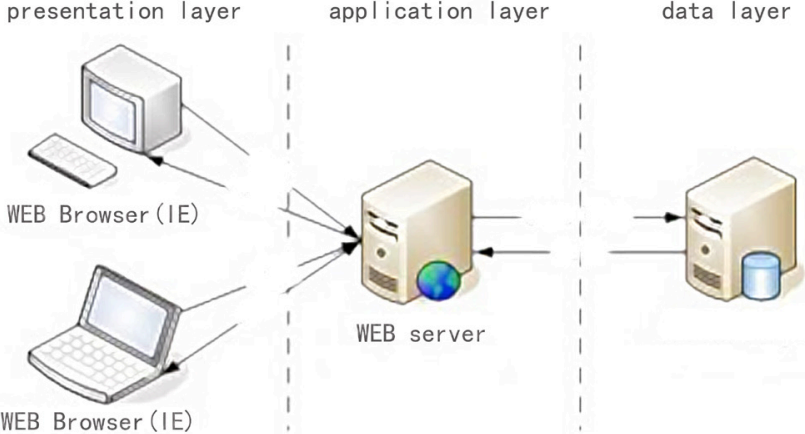

Figure 1. Schematic diagram of $\mathrm{B} / \mathrm{S}$ architectural pattern

The system has a three-layer structure, i.e. database server, WEB server and presentation layer. The data layer is mainly a database server that stores various kinds of data required by the system. The application layer is mainly a WEB server that manages each application module in the system. In the presentation layer, students and teachers can access various kinds of applications in the system through an IE browser in the computer.

\section{2) System module design}

The system includes a student management module, a virtual classroom (interface), a teaching management module, and a learning management module, as shown in Figure 2.

Users use the learning management module to select learning content and to enter the virtual classroom. The student management module interacts with the database server outside the system through the internet to obtain data from other users, generates data from other in-class students, and provides data for the virtual classroom to form an environment of simultaneous learning with mutual competition. The virtual classroom uses the teaching management module for teaching. The users can freely select from five learning forms such as in-class learning, practice, testing, group discussion, and competition to improve their learning. The users can race to be the first to answer questions from virtual teachers, to ask virtual teachers questions, to ask and answer questions mutually and competition with each other. The system can show the performance and level rankings of users in the latest phase and generate special award announcements.

The student management module includes the database server outside the system, a data exchange, a student database, and an in-class student database. The student database conducts data exchange with the database server outside the system through the internet and obtains data from other users. The in-class student database is formed through the combination of content related to the users' learning and the history of the student database. In the system, other users selected by one user do not have to select the user. That is to say, students in the student interface of each user are not necessarily the same, and each user can obtain the data on any other user using this system.

The teaching management module includes a virtual teacher and teaching expert module. Virtual teachers provide teaching materials for students' learning and questions for anticipatory answers, test questions, and PK knowledge. The teaching expert module stores the knowledge base for teaching, including knowledge presented by virtual teachers in class, questions asked to students by virtual teachers, questions asked mutually among students, exercises for students, examination questions for students' tests, knowledge for competition among students, and answers to relevant questions.

The learning management module includes in-class learning, practice, group discussion, and PK. In-class learning, practice, and examination can realize mutual competition among synchronous online users and among

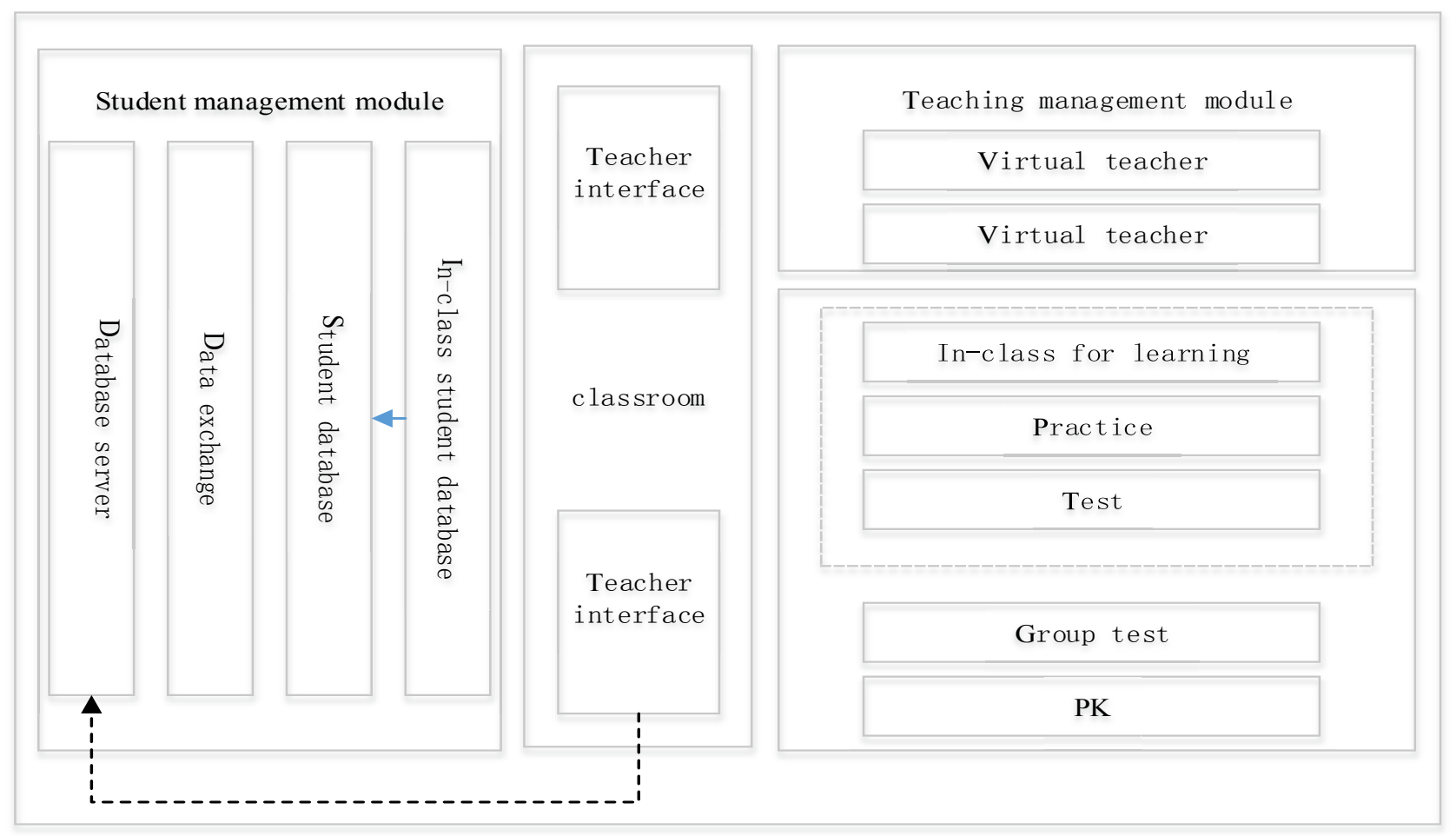

Figure 2. Schematic diagram of system module division 
asynchronous online users. Group discussion and PK are the only mutual actions among the synchronous online users. In-class learning includes listening to the lessons from the virtual teachers, the virtual teachers asking students questions, students asking the virtual teachers questions, and students racing to be the first to answer questions from the virtual teachers. Practice includes virtual teachers specifying exercises in the teaching expert module for students and students asking questions mutually. Examination means that students get a test score ranking through the examination questions provided by teaching expert module. Group discussion is that users form groups for discussion. PK means that students carry out elimination competition through a PK special knowledge base provided by the teaching expert module.

\section{3) Competitive algorithm design}

The synchronous competition mode is simple and is not discussed here. Only in-class question answering, practice, and examination in the asynchronous competition mode are discussed.

In the asynchronous competition mode, when students race to be the first to answer questions from the teachers, the database server records the students' answer and the response time from the question being raised to the students answering, identifies records in the server of other students regarding the same question, retrieves answers in the expert system for comparison, and then ranks correct answers according to the response time.

Under the asynchronous competition mode, when students are doing exercises specified by virtual teachers, the database server records and stores the results of the students after they complete each exercise. When the students ask questions mutually, the students access the question base for mutual question raising in the teaching expert module and then specify another user to answer the question. If this user is offline, students can retrieve his/her records about this question in the database server. If this user is offline and has never answered this question, stu- dents can select another user to try to answer the question until the question is answered. The system database server records each answer of the online students.

In the asynchronous competition mode, students take examinations. The database server records the students' answers, compares their answers with the corresponding answers of the teaching experts, obtains the test result of each student and records it in database server. The database server collects the test results of students in other corresponding examinations and ranks them in descending order.

\section{System realization}

Programming was conducted with the system supporting technologies mentioned above according to its relevant design to realize system functions. Figure 3 is a print screen of a course in the industrial arts major in a virtual classroom system.

\section{EFFECT VERIFICATION}

To verify the availability of a "virtual classroom system under the environment of mutual competition" realized in this paper, the author has applied this system to the industrial arts major and has selected a course for the experiments. The author selected three groups of students to attend this course. The first group of students took lessons traditionally in the school classroom. The second group of students took lessons distantly through video with the method of traditional distance education, and the third group studied through the virtual classroom system designed and realized in this paper. The author designed a group of topics and assessed students respectively from basic knowledge, digital computation, logical reasoning, and abstract theory. Each aspect included 25 questions. There were 100 questions in total, each of which had one score.

For statistics of the average test score of students in the three groups, see Table I.

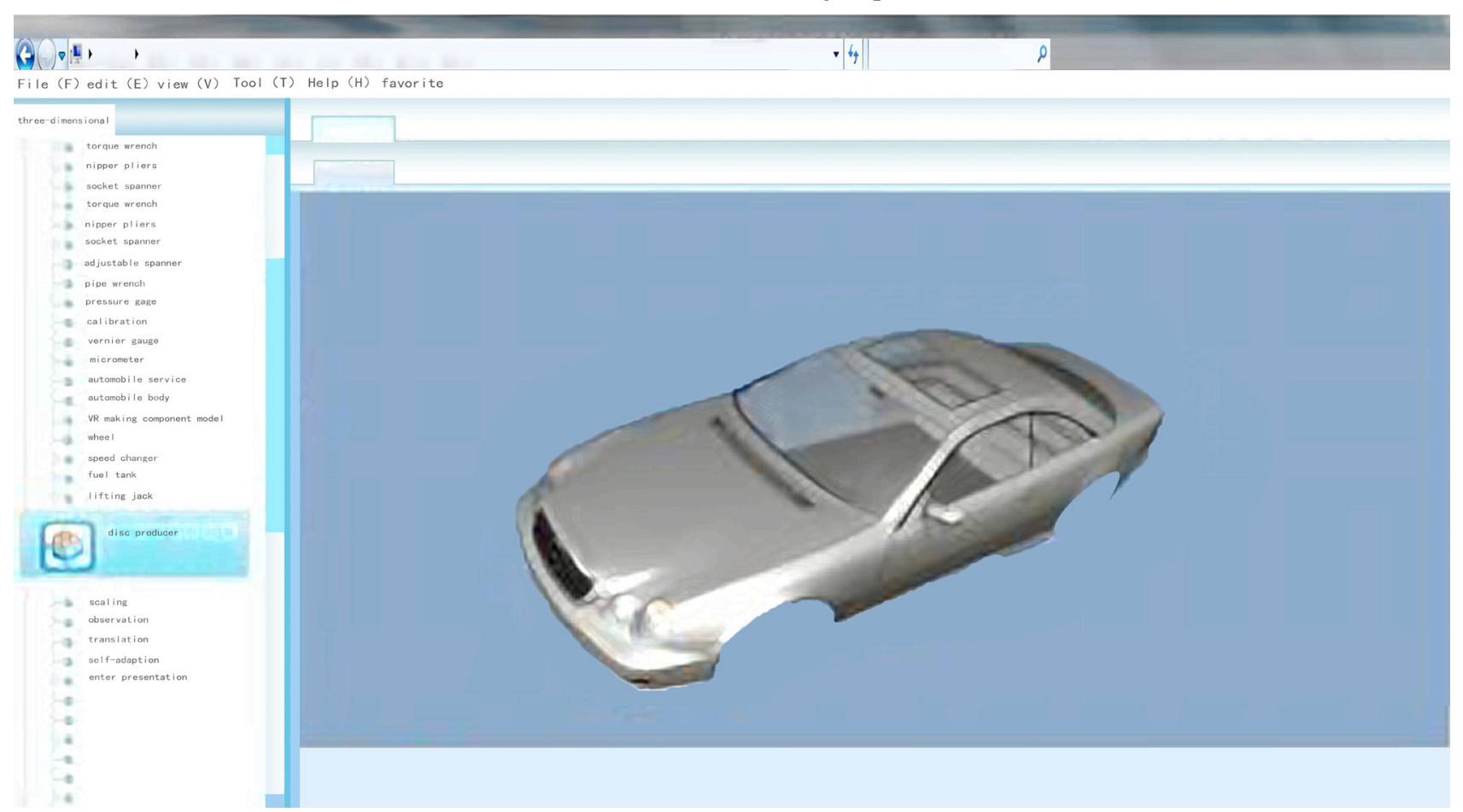

Figure 3. Printscreen of a course in the system 
PAPER

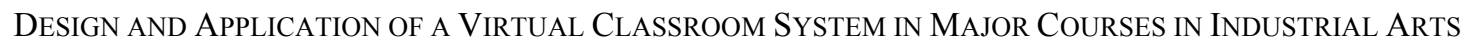

TABLE I.

STATISTICS OF AVERAGE TEST SCORE IN THE EXPERIMENT

\begin{tabular}{lllllr}
\hline \multicolumn{1}{c}{ Student group } & Basic knowledge & Digital computation & Logical reasoning & Abstract theory & Total score \\
\hline Real classroom group & 24 & 24 & 20 & 20 & 88 \\
Distance lessoning group & 21 & 22 & 19 & 18 & 80 \\
Virtual classroom group & 23 & 24 & 22 & 23 & 92 \\
\hline
\end{tabular}

\section{CONCLUSIONS}

Students taking lessons through distance video have lower scores than students in the other two groups because distance video can only provide students with visual and auditory stimulation and does not allow the students to be immersed in a real learning atmosphere. Students taking lessons in a real classroom have scores similar to students taking lessons through virtual classrooms. However, as virtual classrooms can visualize logical reasoning and abstract theory, students in virtual classroom groups can master logical reasoning skills and abstract theoretical knowledge more easily. On the whole, virtual classrooms have a better effect than real classrooms and distance learning.

\section{REFERENCES}

[1] Gary R.M., Gary J.A., "Introduction to the special issue on interaction in distance education," Journal of Computing in Higher Education, vol. 23, no. 2-3, pp. 79-81, December 2011. http://dx.doi.org/10.1007/s12528-011-9050-y

[2] Philip N.C., "Gathering Evidence for Distance Education," Acta de Investigación Psicológica, vol. 4, no. 3, pp. 1657-1672, December 2014.

[3] Maxine L.R, Edmund J.P., "A robust social and professional connection between master educator and Doctor of Nursing Practice (DNP) student instructor: Virtual mentoring and preceptor ship via distance education," Nurse Education Today, vol. 35, no. 5 , pp. 696-699, May 2015. http://dx.doi.org/10.1016/j.nedt. 2015.01.009

[4] Allworth M.B., "Postgraduate distance education in sheep health veterinary education," Small Ruminant Research, vol. 118, no. 13, pp. 97-99, May 2014. http://dx.doi.org/10.1016/j.small rumres.2013.12.013

[5] Weeks K.W., Lyne P., Mosely L., Torrance C., "The strive for clinical effectiveness in medication dosage calculation problem- solving skills: the role of constructivist learning theory in the design of a computer-based 'authentic world' learning environment," Clinical Effectiveness in Nursing, vol. 5, no 1, pp. 18-25, March 2001. http://dx.doi.org/10.1054/cein.2001.0180

[6] Yang K.C., Chang Y.T., Wu C.M., Huang C.M., Wey C.L.. "Universal Learning System for Embedded System Education and Promotion," International Journal of Advanced Computer Science and Applications, vol. 4, no. 2, 4-21, April 2013. http://dx.doi.org/10.14569/IJACSA.2013.040203

[7] Fiona F.H.N., Qing Z., Venkata R.T., Abhishek P.A., Brenda E., "Gamification of Education: A Review of Literature," Lecture Notes in Computer Science, vol. 8527, pp. 401-409, 2014. http://dx.doi.org/10.1007/978-3-319-07293-7_39

[8] Timothy P., Kenneth J.S., "Leveraging Strengths and Meeting Demands: The Construction of a Modern and Comprehensive Electronic Reserves Service at Central Michigan University," Journal of Interlibrary Loan, document Delivery \& Electronic Reserve, vol. 22, no. 1, pp. 17-31, January 2012.

[9] Peng Z.H., Wang D., Wang H., Wang W., "Cooperative Iterative Learning Control of Linear Multi-agent Systems with a Dynamic Leader under Directed Topologies," Acta Automatica Sinica, vol. 40, no. 11, pp. 2595-2601, November 2014. http://dx.doi.org/10.1016/S1874-1029(14)60405-5

[10] Sayed, N.A.M., Zayed, H.H., Sharawy, M.I. ARSC: Augmented Reality Student Card. Computers \& Education. 2011, 56(4):10451061. http://dx.doi.org/10.1016/j.compedu.2010.10.019

\section{AUTHORS}

Yajuan Liu (Corresponding author) is a lecturer at the University of Science and Technology Liaoning, Anshan, 114051, China. Her research interests include virtual classrooms and distance education (yajuanliu11@yeah.net)

This paper was supported by Liaoning Provincial Education Science Twelfth Five Year Plan Project [JG15DB172]. Submitted 08 June 2015. Published as resubmitted by the author 30 October 2015. 